\title{
POLICY FOR THE CROSSMARK
}

https://doi.org/10.54079/jpmi.35.1.2983

Editorial board of the Journal of Postgraduate Medical Institute (JPMI) believes in the integrity of published research, as has been reflected in our various publications on the process of research, its integrity and ethics. The editorial board is committed to maintain the content it publishes, and to alert the readers about the changes in the contents, if and when they occur. JPMI provides its forum to the readers for offering responsible alternative opinions and is open to publish any correspondence (Letter to the editor or a reply to the letter) regarding its published content.

As suggested by ICMJE, corrections are needed for errors of fact. In case, any error or misconduct is reported after publication, the Chief editor of JPMI will constitute a Publication \& Research Integrity Committee comprising of neutral referees for complete and fair investigation of the matter and recommendation to update, correct, or retract the content, if such actions are deemed appropriate.

\section{ERRATUM}

An erratum refers to a correction of errors introduced to the article by the journal in editing or production, including errors of omission such as failure to make factual proof corrections requested by authors within the deadline provided by the journal and within the journal's policy.

During proof stage, JPMI sends the final PDF copy to the corresponding author to identify any errors to be corrected by the journal and approval of the final version by the corresponding author before final publication.

Errors identified after publication by authors or readers are corrected in PDF copy of the online version. Errata are generally not published for simple, obvious, typing errors identified after publication, but are published when an apparently simple error is significant (for example, a Greek - for an ' $\mathrm{m}$ ' in a unit, or a typing error in the corresponding author's email address). A significant error in the figure or table is corrected by publication of a new corrected figure or table as an erratum. The figure or table is republished only if the editor considers it necessary.

\section{CORRIGENDUM}

A corrigendum refers to a change to an article that the author of that article wishes to publish at any time after acceptance. Corrigenda submitted by the original authors are published if the scientific accuracy or reproducibility of the original paper is compromised. JPMI will publish corrigenda if there is an error in the published author list, but not usually for overlooked acknowledgements. Authors should contact the Chief editor JPMI, who will determine the impact of the change and decide on the appropriate course of action. 
Readers wishing to draw the journal's attention to a significant published error, should submit their comments as a "Letter to the Editor". Such "Letters to the Editor" will be carefully reviewed by unrelated and neutral referees. On editorial acceptance, the paper will be sent to the authors of the original paper to provide them with an opportunity for an early response.

\section{ADDENDA}

Addenda are judged on the significance of the addition to the interpretation of the original publication. Addenda do not contradict the original publication, but if the authors inadvertently omitted significant information available to them at the time, this material will be published as an addendum after peer review.

\section{EXPRESSIONS OF CONCERN}

JPMI may consider issuing an Expression of Concern (EOC) if editors have well-founded concerns and feel that readers should be made aware of potentially misleading information contained in an article.

JPMI will consider an expression of concern if:

* They receive inconclusive evidence of research or publication misconduct by the authors.

* There is evidence that the findings are unreliable, but the authors' institution will not investigate the case

* They believe that an investigation into alleged misconduct related to the publication either has not been, or would not be, fair and impartial or conclusive

* An investigation is underway, but a judgement will not be available for a considerable time

\section{RETRACTIONS}

Papers having serious errors to invalidate a paper's results and conclusions, or have issues of publication misconduct, may require retraction. Retractions may be requested by an article's author(s), by an institution, by readers, or by the editor.

As per COPE retraction guidelines, JPMI will consider retracting a publication if:

* There is clear evidence that the findings are unreliable, either as a result of major error (e.g., miscalculation or experimental error), or as a result of fabrication (e.g., of data) or falsification (e.g., image manipulation)

* It constitutes plagiarism

* The findings have previously been published elsewhere without proper attribution to previous sources or disclosure to the editor, permission to republish, or justification (i.e., cases of redundant publication)

* It contains material or data without authorisation for use

* Copyright has been infringed or there is some other serious legal issue

* It reports unethical research 
* It has been published solely on the basis of a compromised or manipulated peer review process

* The author(s) failed to disclose a major competing interest (conflict of interest) that, in the view of the editor, would have unduly affected interpretations of the work or recommendations by editors and peer reviewers.

At times, the article may occasionally be retracted for correction of errors in submission or publication and will be replaced with the corrected one.

\section{RETRACTION PROCESS}

JPMI adopts the following retraction process to ensure best practice of retraction:

An article requiring potential retraction is brought to the attention of Chief Editor JPMI. $\mathrm{He} /$ she should follow the step-by-step guidelines according to the COPE flowcharts and will seek the response from the author of the article as well.

JPMI Publication \& Research Integrity Committee will evaluate the evidence of the misconduct and response of the authors. Based on the findings, committee will recommend a final decision, whether to retract the publication or otherwise.

The final decision is then communicated to the author and, if necessary, any other relevant bodies, such as the author's institution as deemed appropriate.

The retraction-note titled "Retraction: [article title]" will be published with its own DOI, in the paginated part of a subsequent issue of the journal and listed in the contents list. The text of the retraction should explain why the article is being retracted.

The statement of retraction and the original article must be clearly linked in the electronic database so that the retraction will always be apparent to anyone who comes across the original article.

The relevant changes in the online version will be reflected through CrossMark icon.

\section{CROSSMARK}

Crossmark is a multi-publisher initiative from Crossref to provide a standard way for readers to locate the current version of a piece of content. By applying the Crossmark logo, JPMI is committing to maintaining the content it publishes, and to alerting readers to changes if and when they occur.

Clicking on the Crossmark icon will show the current status of a document and may also provide additional publication record information about the document.

Crossmark status will be kept as current, if:

* There are minor changes(like correcting formatting and spelling), with no substantive changes to alert the reader

* The changes don't affect the crediting or interpretation of the work, 
* The changes between versions of a work don't reflect major changes in the content of the paper

Crossmark status of the work will be affected in case of major substantial changes like:

* The retraction of an article due to an error, or

* The changes between versions of a work does reflect major changes in the content of the paper

* A correction to an author's name.

The JPMI content that will have the CrossMark icon is restricted to current and future journal content. 